\title{
A letter from the Director of the African Centre for Disaster Studies - The different faces of vulnerability: Are we making any impact at all?
}

Dewald van Niekerk

Vulnerability is the common cause of the immense disaster risk present on the African continent. For decades the international community has been speculating about the causal and underlying factors which render communities vulnerable to the impact of disasters. In most instances the focus was on the hazardous impact of an event and the surrounding inability of the stricken to deal with the consequences. This emphasis on natural disaster reduction and hazards is highlighted by the objectives of the International Decade of Natural Disaster Reduction (IDNDR - from 1990-1999) which aimed to:

i. Improve the capacity of each country to mitigate the effects of natural disasters, paying special attention to assisting developing countries in the assessment of disaster damage potential and in the establishment of early warning systems and disaster-resistant structures when and where needed;

ii. Develop appropriate guidelines and strategies for applying existing scientific and technical knowledge, taking into account the cultural and economic diversity of different countries;

iii. Foster scientific and engineering endeavours aimed at closing critical gaps in knowledge in order to reduce the loss of life and property;

iv. Disseminate existing and new technological information related to measures for the assessment, prediction and mitigation of natural disasters; and

v. Develop measures for the assessment, prediction and mitigation of natural disasters through programmes of technical assistance and technology transfer, demonstration projects, and education and training, tailored to specific disasters and locations, and to evaluate the effectiveness of those programmes (UN, 1987). 
The emphasis on technologically driven solutions to disaster risk is evident in the above objectives. These ideals were of cause not realised in full and in some instances our communities in Africa are more vulnerable than ever before.

As the successor to the IDNDR, the International Strategy for Disaster Reduction (ISDR) proceeded with the emphasis of the protection against hazards, reducing vulnerability and building resilient communities. The most significant progress that the ISDR aims to make, vis-à-vis the ideals of the IDNDR, is the cultivation of a multidisciplinary approach to disaster reduction within the broader context of sustainable development (UN, 2002). To this end, the Hyogo Framework for Action (UN, 2005) indicates one of its "key activities" as the development of: "... systems of indicators of disaster risk and vulnerability at national and sub-national scales that will enable decision-makers to assess the impact of disasters on social, economic, and environmental conditions and disseminate the results to decision-makers, the public and populations at risk".

Today, after numerous devastating world disasters, we have a much better understanding of the intricacies of disaster risk and how it manifests at community level. We, as a global community, have come to the realisation that community vulnerability cannot be assessed though a macro approach but needs the involvement of the so called "affected". Determining vulnerability must be locally based and locally relevant. In saying this it must be appreciated that, in order to understand and measure vulnerability, a multi-disciplinary approach is needed where the community is the main focal point.

A recent study by the United Nations University on the various components of risk indicates 36 different definitions of vulnerability (Thywissen, 2006). Birkmann (2005) notes that: "We are dealing with a paradox: we aim to measure vulnerability but we cannot define it precisely". The most commonly used definition for vulnerability stems from the ISDR (2004) report "Living with Risk" which defines vulnerability as: "The conditions determined by physical, social, economic and environmental factors or processes, which increase the susceptibility of a community to the impact of hazards". This definition therefore identifies four factors which we need to consider in determining who and what is vulnerable. 
This first edition of Jàmbá aims to focus on some of these different factors and aspects which constitute vulnerability and the research which aims to provide us with some answers and insights into the vulnerability phenomenon. The first paper of this edition by Prof. Djillali Benouar considers the need for an integrated disaster risk management strategy for urban centres with particular reference to the city of Algiers, Algeria. In his paper he makes reference to the multi-layered physical vulnerability which we as human beings create within our urban centres. He postulates that urban risk is the creation of our development and that we need a concerted effort and reorganisation to ensure vulnerability reduction takes place.

Mr. Koos van Zyl takes us away from the urban setting and considers the reduction of agricultural risk though vulnerability assessment. In his paper he considers the different factors which increase vulnerability in farming communities and how "traditional resilience" contributes to vulnerability reduction through local and age-old coping mechanisms. He indicates that vulnerability reduction should enjoy top priority in the various commodity groupings in the agricultural sector.

In the third paper in this edition, Dia, Kouame, Rudant \& Wade presents their technical research in utilising remote sensing technology in order to map flood extension in the Senegal River Estuary near the city of Saint-Louis in Senegal. In their research Dia et al, makes use of various Landsat images and though geometric correction and image fusion manipulates data to provide rich and innovative flood vulnerability maps. Dia et al, proves that remote sensing is a viable option towards the utilisation of leapfrog technology for the African continent.

In staying with geo-spatial technologies, Mr. Dawie van Vuuren illustrates how the use of simple geo-information technologies can be applied to rapid impact assessments following certain hazardous impacts. By using the July 2006 floods in the southern Cape of South Africa as a case study, he indicates that rapid impact assessment must consider the inherent vulnerability present before and after a disaster. His paper indicates that data management and the presence of well structured geo-references data is the cornerstone of successful GIS application in disaster risk management. Mr. van Vuuren's article illustrates how a disaster risk 
management GIS project can easily and quickly be developed in order to rapidly assess vulnerability following a hazardous impact.

In the fifth paper in Jàmbá, Mr. Gideon van Riet focuses on the incorporation of HIV/AIDS considerations in vulnerability assessment for disaster risk reduction. He shows the clear link between HIV/AIDS and vulnerability and how the myriad impacts of HIV/AIDS in any given community adds to an existing volatile risk profile. Mr. van Riet goes on to argue that certain HIV/AIDS proxy indicators used by nongovernmental organisations to target aid during emergency responses can be used as proxy indicators for vulnerability in "normal times". He cautions, however, against the haphazard application of HIV/AIDS indicators in order to determine vulnerability. He concludes by saying that the incorporation of HIV/AIDS considerations into vulnerability assessments is a necessary exercise, as it creates new categories of vulnerable people.

Dr. Guy Sapirstein takes an interesting stance toward social vulnerability and alludes to the importance of addressing social resilience towards vulnerability reduction. $\mathrm{He}$ equates social vulnerability to an economic measurement of vulnerability while social resilience is a factor of risk adaptation over time. He argues that the main principles of a resilience enhancing strategy are: redundancy through overlapping social networks; strengthening response capacities; supporting self-organization before, during and after hazardous events; fostering a culture of learning from past events; and encouraging adaptation to hazardous conditions. He concludes in saying that increasing the strength of a society is about increasing the strength and scope of the internal connections between the people, organizations and environment that form that society. Moving away from the doctrine of independence to embracing a culture of interdependence is the key to both harmony and development.

We trust that you will find this first issue of Jàmbá both interesting and thought provoking. I would like to encourage all academics, researchers, practitioners and senior students on the continent to find time to provide us with your research insights and findings in disaster risk reduction by contributing to Jàmbá. 


\section{References}

BIRKMANN, J. 2005. Danger need not spell disaster, but how vulnerable are we? Research Brief. 1. Germany: UNU-EHS.

INTERNATIONAL STRATEGY FOR DISASTER REDUCTION (ISDR). 2004. Living with risk: A global review of disaster reduction initiatives. Geneva: ISDR Secretariat.

THYWISSEN, K. 2006. Components of risk: A comparative glossary. SOURCE 2/2006. Germany: UNU-EHS.

UNITED NATIONS (UN). 2005. Hyogo Framework for Action 2005-2015: Building the resilience of nations and communities to disasters. Geneva:ISDR.

UNITED NATIONS (UN). 2002. General Assembly Resolution 56/195. International Strategy for Disaster Reduction. 21 January. Geneva: UN.

UNITED NATIONS (UN). 1987. General Assembly Resolution 42/169. The International Decade for Natural Disaster Reduction. 3 February. Geneva: UN. 